\title{
TEV-3349 "ATF-2 2A Loop Pin Cladding Requirements"
}

\author{
Brian P Durtschi
}

February 2020

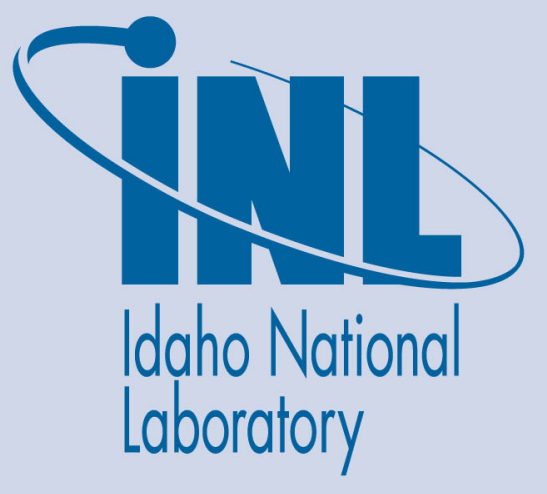

The INL is a U.S. Department of Energy National Laboratory operated by Battelle Energy Alliance 


\title{
TEV-3349 "ATF-2 2A Loop Pin Cladding Requirements"
}

\author{
Brian P Durtschi \\ February 2020 \\ Idaho National Laboratory \\ Idaho Falls, Idaho 83415 \\ http://www.inl.gov \\ Prepared for the \\ U.S. Department of Energy \\ Under DOE Idaho Operations Office \\ Contract DE-AC07-05ID14517
}




\begin{tabular}{|c|c|}
\hline 1. Effective Date & ---- \\
\hline $\begin{array}{l}\text { 2. Does this ECAR involve a } \\
\text { Safety SSC? }\end{array}$ & No \\
\hline $\begin{array}{l}\text { 3. Safety SSC Determination } \\
\text { Document ID }\end{array}$ & $\mathrm{N} / \mathrm{A}$ \\
\hline 4. SSC ID & $\mathrm{N} / \mathrm{A}$ \\
\hline 5. Project No. & 31418 \\
\hline 6. Engineering Job (EJ) No. & N/A \\
\hline 7. Building & Multiple Site Areas \\
\hline 8. Site Area & Multiple Site Areas \\
\hline 9. CUI Determination & No CUI \\
\hline
\end{tabular}

Professional Engineer's Stamp

(Identify whether the use of a registered professional engineer is required.

See LWP-10010 for requirements

For example: No, per Sec 4.1, par. aa)

10. Objective / Purpose

Commercial fuel pins are fabricated by putting cylindrical fuel pellets in tubular cladding with welded end plugs. They are fabricated per industry proprietary processes as opposed to code standards. As such, ASME BPVC code is not applicable in this instance because of the unique materials that are used for cladding and because of the geometry of the system, in that these are sealed capsules, not pressure vessels with attendant piping.

In support of the ATF-2 series of irradiation tests, a variety of fueled test specimens (rodlets) are fabricated for irradiation testing in the 2A loop of ATR. All these rodlets consist of cladding around cylindrical fuel pellets but vary in configuration and composition. Metallic rodlets are fabricated both at INL and at industry partner facilities. Silicon Carbide clad rodlets are fabricated by industry partners.

There are two drivers and sets of requirements to ensure the structural integrity of the fuel pin cladding; SAR/ESA/safety, and programmatic.

Demonstration that the SAR/ESA/safety requirements are met can be accomplished, via proof testing in the loop, which is explained in Appendix $B$ in addition to the analysis and fabrication documentation contained in the ESA.

The programmatic requirements will be met using the appendices applicable to that particular pin and fabrication location. These will have rigor derived from adherence to the theories that were used to construct the American Society of Mechanical Engineers Boiler and Pressure Vessel Code (ASME BPVC) but the fuel pins do not meet the code or equivalent code requirements.

Both the safety and the applicable programmatic requirements will be followed for the pins constructed for the ATF-2 program, as designated in the appropriate appendices. 
11. If revision, please state the reason and list sections and/or page being affected.

Entire document revised. The scope was decreased to only include ATF-2 pins that will be irradiated in the center loop. Differences between requirements for safety and for programmatic concerns are more clearly delineated. Edited for clarity and to move to the new template.

The safety requirements listed in Appendix B should be adhered to for all pins as required by the ESA. The programmatic requirements listed in the related appendix will be followed for the remaining pins. 


\section{CONTENTS}

PROJECT ROLES AND RESPONSIBILITIES.............................................. 4

SCOPE AND BRIEF DESCRIPTION...................................................... 5

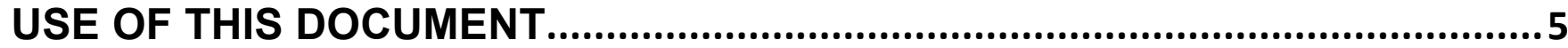

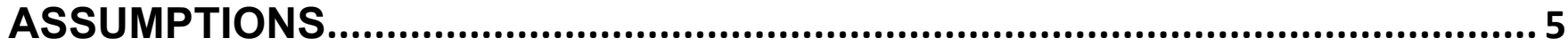

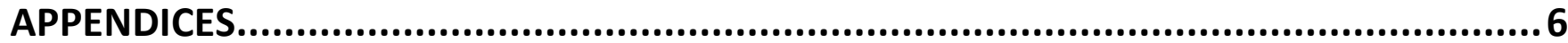

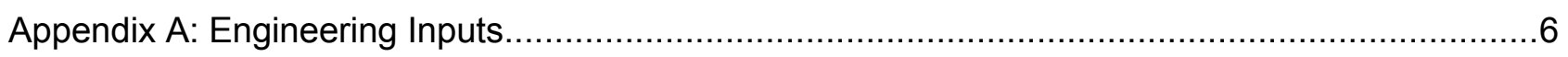

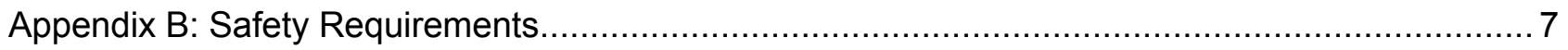

Appendix C: Load Conditions...................................................................................... 8

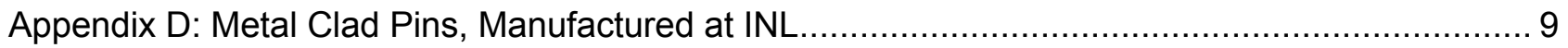

Appendix E: Metal Clad Pins, Manufactured by Industry Partners............................................. 12

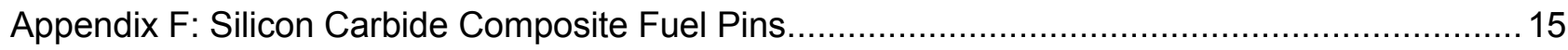




\section{PROJECT ROLES AND RESPONSIBILITIES}

\begin{tabular}{|c|c|c|c|}
\hline Project Role & Name & Organization & Pages Covered (if applicable) \\
\hline Performer & Brian Durtschi & C660 & $\begin{array}{ll}---- \\
\end{array}$ \\
\hline Checker $^{\mathrm{a}}$ & Connor Woolum & U110 & ----- \\
\hline Independent Reviewer ${ }^{b}$ & Stephen Evans & G520 & $\begin{array}{l}---- \\
--1\end{array}$ \\
\hline CUI Reviewer ${ }^{c}$ & Brian Durtschi & C660 & ----- \\
\hline Manager $^{d}$ & Doug Stacey & C660 & 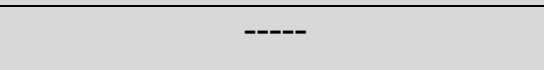 \\
\hline Requestor $^{\mathrm{ef}}$ & David Kamerman & C620 & ----- \\
\hline Nuclear Safety ${ }^{f}$ & Kirk Stueve & G000 & ----- \\
\hline Document Owner $^{f}$ & David Kamerman & C620 & ----- \\
\hline Reviewer ${ }^{f}$ & David Kamerman & C620 & $\begin{array}{ll}---- \\
--1\end{array}$ \\
\hline
\end{tabular}

\section{Responsibilities:}

a. Confirmation of completeness, mathematical accuracy, and correctness of data and appropriateness of assumptions.

b. Concurrence of method or approach. See definition, LWP-10106.

C. Concurrence with the document's markings in accordance with LWP-11202.

d. Concurrence of procedure compliance. Concurrence with method/approach and conclusion.

e. Authorizes the commencement of work of the engineering deliverable. See Appendix A.

f. Concurrence with the document's assumptions and input information. See definition of Acceptance, LWP10200. A system under which it takes three men to check what one is doing is not control; it is systematic strangulation. H. Rickover.

NOTE: Delete or mark "N/A" for project roles not engaged. Include ALL personnel and their roles listed above in the eCR system. The list of the roles above is not all inclusive. If needed, the list can be extended or reduced. 


\section{SCOPE AND BRIEF DESCRIPTION}

Commercial fuel pins are fabricated by putting cylindrical fuel pellets in tubular cladding with welded end plugs. They are fabricated per industry proprietary processes as opposed to code standards. Similarly, ASME BPVC code is not applicable in this instance because of the unique materials that are used for cladding and because of the geometry of the system, in that these are sealed capsules, not pressure vessels with attendant piping.

In support of the ATF-2 series of irradiation tests, a variety of fueled test specimens (rodlets) are fabricated for irradiation testing in the 2A loop of ATR. All these rodlets consist of cladding around cylindrical fuel pellets but vary in configuration and composition. Metallic rodlets are fabricated both at INL and at industry partner facilities. Silicon Carbide clad rodlets are fabricated by industry partners.

There are two drivers and sets of requirements to ensure the structural integrity of the fuel pin cladding; SAR/ESA/safety, and programmatic.

Demonstration that the SAR/ESA/safety requirements are met can be accomplished, via proof testing in the loop, which is explained in Appendix $B$ in addition to the analysis and fabrication documentation contained in the ESA.

The programmatic requirements will be met using the appendices applicable to that particular pin and fabrication location. These will have rigor derived from adherence to the theories that were used to construct the American Society of Mechanical Engineers Boiler and Pressure Vessel Code (ASME BPVC) but the fuel pins do not meet the code or equivalent code requirements.

Both the safety and the applicable programmatic requirements will be followed for the pins constructed for the ATF-2 program, as designated in the appropriate appendices.

\section{USE OF THIS DOCUMENT}

The safety requirements listed in Appendix B, "Safety Proof Testing", shall be met for all pins as required by the ESA.

The programmatic requirements shall be found in the individual appendix related to those individual pins.

\section{ASSUMPTIONS}

1. The fuel pins fabricated and tested under these guidelines will be irradiated in the center loop of the ATR reactor.

2. The pins will be moved per established procedures from the reactor to the canal and in the canal. 


\section{APPENDICES}

\section{Appendix A: Engineering Inputs}

This TEV is required to show how the ATF-2 program will provide an acceptable level of rigor for the ATF-2 fuel pins that will be irradiated in the 2A Center Position Loop. 


\section{Appendix B: Safety Requirements}

Proof testing can be used by the Experiment Safety Analysis (ESA) to demonstrate compliance with SAR-153 safety requirements. The following shall be performed on all pins as required by the ESA.

Proof testing on ATF-2 pins after irradiation will be performed in the loop prior to removal from the in-pile tube. The proof testing will consist of the following steps

1. Maintain forced flow cooling in the loop 24 hours minimum.

2. Take baseline radiation measurements using loop radiation instruments and take a BIX sample.

3. Shut off loop pumps and depressurize the loop for a minimum of 1 hour.

4. Re-pressurize and run the loop pumps for 30 minutes.

5. Observe radiation instruments and contact engineering, Radcon, and shift supervisor for resolution if radiation levels increase.

6. If any radiation increases are noted take a BIX sample and have RML perform isotopic analysis of the baseline sample and the sample taken after the proof test. Evaluate the fission product isotopes to confirm a fission product release has not occurred.

7. If no radiation level increases are detected, the cladding is assumed to be intact and failure probabilities outside of the in-pile tube are assumed to occur at a lower frequency.

Testing the fuel pins in the in-pile tube demonstrates that fuel pin failure does not occur under higher heat loads and less natural convection than present during subsequent handling and storage evolutions. Decay heat from the fuel pins will be highest immediately following shutdown while the experiment is still in the in-pile tube. Additionally, the experiment experiences gamma heating from the reactor and is surrounded by smaller flow channels while inside the in-pile tube.

Increases in the fuel loading over the analyzed condition or significant changes in the flow path will require recalculation of required time of step 3 . This calculation is based on the ATF-2 safety case, used for the first insertion. 


\section{Appendix C: Load Conditions}

\section{Load Case 1:}

This load case simulates the maximum internal pressure for pins pressurized in the WUPS system. After the final seal weld is made and the system is depressurized, the internal pressure will be the pressure difference across the wall.

Internal Pressure $=500 \mathrm{psi}$

Maximum WUPS system pressure is used

Material Temperature $=70^{\circ} \mathrm{F}$

Room temperature was chosen

\section{Load Case 2:}

After the pins are irradiated in the ATR, the pins will be placed in the canal. This load case simulates the maximum fission gas release pressure and the condition where coolant is still available. This covers the period while in the transfer cask, in the loop depressurized, and in canal storage.

Internal Pressure, From Physics Analysis, EOL,

The internal pressure will use an internal temperature of $300^{\circ} \mathrm{F}$. The fuel pin internal area has both a plenum area and an annulus area between the fuel and the cladding. The annulus is very small relative to the plenum. The temperature driving the internal gas will be the coolant temperature.

Cladding Temperature $=300^{\circ} \mathrm{F}$, or as shown by calculation

All numbers used for analysis will be confirmed using physics and thermal analysis.

\section{Other Load Conditions}

Other load conditions, such as shipping after irradiation, shall be analyzed and documented in shipping ECARS. 


\section{Appendix D: Metal Clad Pins, Manufactured at INL}

\section{Basis}

Instead of making up a basis from scratch and possibly missing important concepts, we will consider our requirements against ASME BPVC Section III. ASME BPVC does not form the actual requirements, but gives items for consideration in constructing the requirements as laid out in this TEV.

- $\quad$ Material, NB-2000

- $\quad$ Design NB-3000

- $\quad$ Fabrication \& Installation, NB-4000

- Examination NB-5000

- $\quad$ Testing NB-6000

- $\quad$ Overpressure Protection NB-7000

\section{Materials}

All non-irradiated pressure retaining materials shall have known chemistry and material properties. These items shall be provided either through the supplier or through testing done by the INL. Any supplying of parts to INL by industry partners will be according to their quality program, which will be verified per INL quality requirements. The procurements of the materials and testing services from other vendors shall be performed per the INL quality manual.

\section{Design and Analysis}

A design-by-analysis approach shall be followed as a guide in accordance with ASME BPVC Section III, Division 1 as required. If a simplified approach to analysis is taken, justification for deviation from the code shall be documented. Thermal and structural analyses shall be performed, documented in a technical report, such as an Engineering Calculations and Analysis Report (ECAR), and checked by a qualified peer in the relevant discipline. Technical reports shall be used to verify design in place of a "certified design report." The material properties designated in the materials section above shall be used. In the ASME BPVC, Section III, Div. 1, Subsection NB (Class 1 Components), the basic stress limit is called the Design Stress Intensity, $\mathrm{S}_{\mathrm{m}}$. The Design Stress Intensity shall be determined using the method detailed in Section II, Part D, Mandatory Appendix 2. Cladding that is subject to temperatures where time-dependent material properties are applicable shall follow as a guide the rules of Subsection NH (Class 1 Components in Elevated Temperature Service) or other applicable material-specific methods. Part tolerances and marking depths will be taken into account in any locations where stresses are of concern.

This analysis specifically considers conditions when the pins are not enclosed in the in pile tube. It does not include thermal stress ratcheting (cycle numbers are low) or fatigue failure (low number of cycles and large loads are compressive.

The performer and/or the checker of these calculations shall have ASME Section III experience as deemed 
appropriate by their management. An ASME code symbol stamp shall not be required for the component.

Strengths of irradiated materials may be taken from the appropriate literature by the principal investigator.

The load configurations/design conditions to be analyzed are found in Appendix C: "Load Conditions".

\section{Fabrication and Assembly}

Fabrication performed at INL shall be performed using approved INL procedures applicable to the purpose. Corresponding INL quality procedures shall be followed. Fabrication services performed by non INL sources shall be acquired and verified per INL quality procedures. If machining besides cutting to length and preparing the end weld surfaces is performed on the cladding tube, all accessible tube surfaces shall be examined using dye penetrant testing per ASME BPVC Section V Article 6 with acceptance criteria per ASME BPVC Section III NB-2546.3.

All welding performed on non-irradiated materials shall conform to ASME Section IX and shall be performed in accordance with INL weld procedures.

\section{Examination and Inspection}

\section{Dimensional Verification}

Critical dimensions such as wall thickness and diameter shall be verified per approved quality procedures. The tube is the location of the aggressive service (hottest) highest flux, greatest corrosion risks, greatest through wall thermal stresses, and cladding fuel interactions. The cladding must be checked to minimize risks due to walls that are too thin or too thick. The tube is being singled out as the dimensions in the cap are only critical to the weld fitup, which will be checked as part of the welding process. These dimensions will be measured prior to the application of any coating material.

If the tube is produced via machining:

1. Verification measurements shall be performed on every piece of tube.

2. Wall thickness measurements shall be made a minimum of three axial locations, including each end and at the center.

3. At each axial location, thickness measurements will be made of two locations located 90 degrees from each other.

4. The inspection process shall be clearly identified, appropriately documented, and reviewed during the design review to ensure the requested inspections are sufficient.

If the tube is produced via drawing, pilgering, extrusion, or other similar continuous process:

1. Continuous measurements that check minimum wall thickness during manufacture will be suitable, provided they demonstrate the tube is withing drawing specification. In the case of a commercial fuel pin cladding, meeting the commercial fuel pin cladding specification shall be considered sufficient.

2. If continuous measurements were not made, the requirements directly above for wall verification of machined cladding may be used. 


\begin{tabular}{|lrr|}
\hline TEM-10300-1, Rev. 7 & TECHNICAL EVALUATION & TEV- 3349, Rev. 1 \\
$01 / 29 / 2020$ & Page $\mathbf{1 3}$ of $\mathbf{2}$ \\
& ATF-2 2A Loop Pin Cladding Requirements & \\
\hline
\end{tabular}

\section{Post welding verifications}

\section{Hermeticity-}

Helium Leak check shall be performed on all fuel pins per ASME BPVC Section V Article 10 Mandatory Appendix V. Acceptance criteria shall be a maximum leak rate of $1 \times 10^{-7} \mathrm{Std}$ cc/second.

\section{Surface Flaw Inspections}

Visual examination will be performed on all pin pressure boundary welds, with acceptance criteria of no linear indications or visible porosity.

Weld penetrant examination shall be performed on all metallic pressure boundary welds on non-irradiated specimens per ASME BPVC Section V Article 6 with acceptance criteria per ASME BPVC Section III NB-5352.

\section{Volumetric Inspections}

Radiography shall be performed on all pressure boundary welds per ASME BPVC Section V with acceptance criteria per ASME BPVC Section III NB-5320.

\section{Overpressure Protection}

These rodlets are small volumes that are intentionally sealed without any openings, and it would be nonsensical to have a pressure relief valve. There will be no overpressure protection. 


\section{Appendix E: Metal Clad Pins, Manufactured by Industry Partners}

\section{Basis}

Instead of making up a basis from scratch and possibly missing important concepts, we will consider our requirements against ASME BPVC Section III. ASME BPVC does not form the actual requirements, but gives items for consideration in constructing the requirements as laid out in this TEV.

- $\quad$ Material, NB-2000

- $\quad$ Design NB-3000

- $\quad$ Fabrication \& Installation, NB-4000

- $\quad$ Examination NB-5000

- $\quad$ Testing NB-6000

- $\quad$ Overpressure Protection NB-7000

\section{Materials}

All non-irradiated pressure retaining materials shall have known chemistry and material properties. The documentation of these materials shall be sent to the INL in as built packages per vendor quality programs. INL quality will verify vendor quality programs per INL procedures.

\section{Design and Analysis}

This analysis specifically considers conditions when the pins are not enclosed in the in pile tube. It does not include thermal stress ratcheting (cycle numbers are low) or fatigue failure (low number of cycles and large loads are compressive). Analysis of the pins in the PWR environment shall be performed by the vendors, per their very well developed proprietary methods per their quality program.

A design-by-analysis approach shall be followed as a guide in accordance with ASME BPVC Section III, Division 1 as required. If a simplified approach to analysis is taken, justification for deviation from the code shall be documented. Thermal and structural analyses shall be performed, documented in a technical report, such as an Engineering Calculations and Analysis Report (ECAR), and checked by a qualified peer in the relevant discipline. Technical reports shall be used to verify design in place of a "certified design report." The material properties designated in the materials section above shall be used. In the ASME BPVC, Section III, Div. 1, Subsection NB (Class 1 Components), the basic stress limit is called the Design Stress Intensity, $\mathrm{S}_{\mathrm{m}}$. The Design Stress Intensity shall be determined using the method detailed in Section II, Part D, Mandatory Appendix 2. Cladding that is subject to temperatures where time-dependent material properties are applicable shall follow as a guide the rules of Subsection NH (Class 1 Components in Elevated Temperature Service) or other applicable material-specific methods. Part tolerances and marking depths will be taken into account in any locations where stresses are of concern.

The performer and/or the checker of these calculations shall have ASME Section III experience as deemed appropriate by their management. An ASME code symbol stamp shall not be required for the component. 
Strengths of irradiated materials may be taken from the appropriate literature by the principal investigator.

The load configurations/design conditions to be analyzed are found in Appendix C: "Load Conditions".

\section{Fabrication and Assembly}

Any welding performed outside the INL shall have a welding process that is qualified per vendor quality program, and proof shall be provided to the INL quality program. If machining besides cutting to length and preparing the end weld surfaces is performed on the cladding tube, all accessible tube surfaces shall be examined using dye penetrant testing per ASME BPVC Section V Article 6 with acceptance criteria per ASME BPVC Section III NB2546.3 .

All fabrication and parts tracking shall occur per vendor quality program, which will be verified per INL quality procedures.

\section{Examination and Inspection}

\section{Dimensional Verification}

Critical dimensions such as wall thickness and diameter shall be verified per approved quality procedures. The tube is the location of the aggressive service (hottest) highest flux, greatest corrosion risks, greatest through wall thermal stresses, and cladding fuel interactions. The cladding must be checked to minimize risks due to walls that are too thin or too thick. The tube is being singled out as the dimensions in the cap are only critical to the weld fitup, which will be checked as part of the welding process. These dimensions will be measured prior to the application of any coating material.

If the tube is produced via machining:

1. Verification measurements shall be performed on every piece of tube.

2. Wall thickness measurements shall be made a minimum of three axial locations, including each end and at the center.

3. At each axial location, thickness measurements will be made of two locations located 90 degrees from each other.

4. The inspection process shall be clearly identified, appropriately documented, and reviewed during the design review to ensure the requested inspections are sufficient.

If the tube is produced via drawing, pilgering, extrusion, or other similar continuous process:

1. Continuous measurements that check minimum wall thickness during manufacture will be suitable, provided they demonstrate the tube is withing drawing specification. In the case of a commercial fuel pin cladding, meeting the commercial fuel pin cladding specification shall be considered sufficient.

2. If continuous measurements were not made, the requirements directly above for wall verification of machined cladding may be used 


\section{Post welding verifications}

\section{Hermeticity-}

Helium Leak check shall be performed on all fuel pins per ASME BPVC Section V Article 10 Mandatory Appendix V. Acceptance criteria shall be a maximum leak rate of $1 \times 10^{-7} \mathrm{Std} \mathrm{cc} / \mathrm{second}$.

\section{Surface Flaw Inspections}

Visual examination will be performed on all pin pressure boundary welds, with acceptance criteria of no linear indications or visible porosity. Commercial fuel pin fabrication specifications do not require dye penetrant inspection. Since these pins are being fabricated in the same manner as commercial fuel pins, dye penetrant inspection is not required.

\section{Volumetric Inspections}

Industry partners have machinery and procedures designed to produce large quantities of fuel pins. These pins are produced via their commercial equipment and processes, such as resistance flash welding. These processes and equipment are extremely consistent for a given weld geometry. If cladding materials are of the same chemistry and geometry as the industry partner's commercial cladding, and if they are welded in the same manner, they may use the same process they use for commercial fuel pins.

For fuel pins with cladding made of novel materials and manufactured by commercial fuel vendors using resistance flash welding, one of two methods of inspection may be used; volumetric examination or destructive testing of test articles.

Radiography shall be performed on all pressure boundary welds performed per ASME BPVC Section V with acceptance criteria per ASME BPVC Section III NB-5320.

If destructive testing of test articles is used, all welds from a given batch will be performed sequentially with the same set up and equipment in one day. The first and last welds performed from each batch will be used be used for metallography samples. Any failure of a weld to meet design criteria in any sample renders the entire batch failed. The sample pins will be identical to the fuel pins in cladding and contents.

\section{Overpressure Protection}

These rodlets are small volumes that are intentionally sealed without any openings, and it would be nonsensical to have a pressure relief valve. There will be no overpressure protection. 


\section{Appendix F: Silicon Carbide Composite Fuel Pins}

\section{Overview}

Silicon carbide pins will be provided by industry partners. These pins are composed of a silicon carbide fiber weave that is impregnated with a solid silicon carbide. These composites are in some ways similar to solid ceramics but exhibit a pseudo-ductility as well. They also have a temperature dependent swelling mechanism under irradiation. These differences make analysis of silicon carbide pins different than metal pins and require changes to requirements for metal pins; however, high temperature strength, corrosion performance, and neutronic performance makes these pins excellent candidates for accident tolerant fuels and for high temperature reactors.

This revision of TEV-3349 is for silicon carbide pins with surrogate fuel. Actual fuel will require the concurrence of ATR management.

\section{Materials}

All components of silicon carbide pins, including the pressure boundary and the contents, shall have known chemistry and material properties. Documentation of records shall be performed in accordance with the industry partner quality programs. These quality programs will be verified through audit by INL quality personnel.

\section{Design and Analysis}

Dimensions shall comply with INL drawings that are established and will be used for mechanical fit, physics analysis, and thermal analysis.

ASME BPVC does not have the proper material data set to model SiC interaction. SiC has a pseudo-ductility and exhibits significant and temperature dependent swelling early in irradiation. These are not accounted for in the ASME BPVC, and other modeling techniques developed specifically for SiC composites will be used. Industry partners have developed these techniques based on data derived from irradiated and unirradiated samples.

Using the techniques, industry partners shall:

1. Report on cladding stresses predicted for the ATR irradiation conditions. These simulations shall include any ratcheting effects from the start ups and shutdowns.

2. Allowable base hoop and axial composite strengths will be based on witness specimens that are fabricated from the same batch of materials using the same parameters for all special processes. The irradiated properties of materials used in the model will be based on data gathered from previous irradiations and are expected to nearly identical to unnirradiated properties. Irradiation growth, changes in properties over irradiation, and pseudo-ductility shall be considered.

3. Provide a record that demonstrates the fabrication and steps listed above were performed by qualified personnel and performed and documented in accordance with qualified quality program. 


\section{Fabrication and Assembly}

Fabrication shall be performed per qualified procedures established in accordance with industry partner quality programs.

\section{Joining Process}

The joining processes used for $\mathrm{SiC}$ provide a similar function to welding but operate very differently. These processes are proprietary and developed by each industry partner. They are performed with tight control of critical process variables. These variables will be designated and qualified in a procedure, and that procedure shall be complied with for final procedures. The joining will be performed by qualified individuals and will be documented per the industry partner quality program.

\section{Examination and Inspection}

\section{Dimensional Verification}

Verification measurements shall be performed on every piece of tube. The tube is the location of the aggressive service (hottest) highest flux, greatest corrosion risks, greatest through wall thermal stresses, and cladding fuel interactions.

Critical dimensions such as wall thickness and inner and outer diameters shall be verified per approved quality procedures. Due to the possible irregularity of interior surfaces, measurements that can accurately measure the inner surface such as computed tomography will required to verify the dimensions of the tube prior to assembly.

Chemical Compatibility.

Industry partner shall perform representative corrosion tests. These shall be either 1) in reactor tests or 2) flowing autoclave tests on the specimen with chemistry to simulate radiolysis. Testing parameters shall be agreed up per INL engineering. Any material loss rates established by previous testing shall be shown not to affect hermeticity of the fuel pin over the irradiation.

\section{Post joining verifications}

Hermeticity-

Helium Leak check shall be performed on all fuel pins per ASTM E493. Acceptance criteria shall be a maximum leak rate of $1 \times 10^{-7} \mathrm{Std} \mathrm{cc} /$ second.

This shall be performed by industry partners prior to shipping, and then by INL on receipt confirm hermeticity after shipping.

Industry partners shall establish and qualify a process that will be duplicated by the INL upon receipt. This include things such as bombing time and allowable outgas time for bombed specimen, recognizing that outgassing takes significantly longer for $\mathrm{SiC}$ specimens. These methods must be deemed acceptable by INL quality.

\section{Surface Flaw Inspections}

No dye penetrant testing will be required. The surface finish of silicon carbide composite would give to many false positives to be able to discern a true positive result, and the penetrant would not be completely recoverable from the surface. 


\section{Radiography}

Radiography by qualified personnel shall be performed at each joint using procedures qualified by the industry partner per their quality program. This radiography may be either computed tomography or 2D images on all deliverable parts, with a minimum resolution of .001 inches. Images will be taken at a minimum of every 60 degrees.

The acceptance criteria shall be established by industry partners, with INL acceptance. It will show hermiticity and structural integrity will be maintained through full irradiation per analysis and corrosion rate data

\section{Additional witness article testing}

Joint strength will be tested on a minimum of two witness specimens from the same batch of material as the deliverables. This joint testing shall be performed with pressurization or with a test that has been shown to be equivalent to pressurization for the purposes of testing joints made using the same process type. This equivalency may be shown on the same batch or on another batch with similar properties.

\section{Overpressure Protection}

These rodlets are small volumes that are intentionally sealed without any openings, and it would be nonsensical to have a pressure relief valve. There will be no overpressure protection. 